\title{
EDITORIAL
}

\section{In This Issue: Decisions, Decisions}

\author{
Kurt C. Stange, $M D, P b D$, Editor
}

Ann Fam Med 2016;198-199. doi: 10.1370/afm.1948.

$\mathrm{T}$ This issue of Annals sheds light on patient and clinician decisions and their implications.

Hendriksen and colleagues compare family physicians' gestalt (estimated probability) of pulmonary embolism compared to the Wells decision rule among patients referred for testing. Gestalt performs surprisingly well. The Wells rule is more specific, and almost as sensitive as physicians' gestalt, making it more efficient. ${ }^{1}$

An interesting qualitative study by Phillips and colleagues uncovers personal and professional strategies used by women in rural family practice for work-life balance. These strategies involve decisions on multiple levels-from the individual, to personal relationships to building community support for parenting roles. ${ }^{2}$

DeVoe shares how wearing both her daughter and doctor hats brought meaning to her and help to her father in making decisions (and avoiding unhelpful intervention) during the last week of his life. ${ }^{3}$

In navigating an urgent medical decision in the context of a high-deductible insurance plan, being a physician provides little help in uncovering hidden and wildly varied price variation for needed services. ${ }^{4}$

Decisions are framed by context and relationships. A clinical trial by Purkaple et al asks "Can patients help their physicians be patient centered?" Surprisingly, an intervention that asks patients questions about quality of life goals and concerns leads to little focus on quality of life during physician visits, compared to a control intervention that asks about symptoms. Even more startling, directly observed empathy is greater in encounters in the control group. ${ }^{5}$

An article featured in this issue's Annals Journal Club examines the survival expectations of patients with localized prostate cancer detected by screening. Their treatment decisions are affected by patients' overestimating survival with treatment and underestimating survival without treatment. These findings have important implications for how we help patients to make truly informed treatment choices. ${ }^{6,7}$

Among patients who undergo colorectal cancer treatment with curative intent, $58 \%$ of recurrences are detected during scheduled follow-up visits, and $42 \%$ of recurrences are found during non-scheduled interval visits-most of these involving germane symptoms. ${ }^{8}$

An impressively multi-method, multi-perspective study by Valderas et al develops and validates a new measure of Patient Reported Experiences and Outcomes of Safety in Primary Care. ${ }^{9}$ This tool opens up new possibilities for practice improvement and research.

Implementation of a knowledge transfer intervention around the Ottawa Model for Smoking Cessation is associated with greater rates of delivery of smoking cessation treatment, as is the quality of the program implementation. ${ }^{10}$

Having patients measure their own waist circumference after written and pictorial instructions seems like a good idea, but is found to have a high false negative rate for assessing overweight, in a study by Jensen and colleagues. ${ }^{11}$

The duration of effectiveness of a health coaching intervention for chronic disease management is examined by Sharma and colleagues. The results are reassuring of a persistent effect. ${ }^{12}$

An insightful Reflection by Olayiwola shares a personal experience in which a racist rant by a patient seems to reverse the usual power dynamic, but also uncovers tools for resilience. ${ }^{13}$

We are pleased to welcome new members to the Annals team.

Michael Johansen, MD, MS joins as the new Annals Editorial Fellow. Dr Johansen is Assistant Professor in the Department of Family Medicine at The Ohio State University. He teaches evidence-based medicine and the journal club at his family medicine residency, and brings an interest in the critical review of evidence, health policy, and health care delivery research.

Alan Adelman, MD, MS joins us as Senior Editorial Fellow, bringing deep experience in family medicine research. Dr Adelman is the Jeanne L. and Thomas L. Leaman Professor of Family \& Community Medicine at the Penn State University College of Medicine.

We are grateful to our departing Editorial Fellow, Aaron M. Orkin, MD, MSc, MPH who had a strong and forward-reaching impact on Annals. You will hear from him in a forthcoming editorial and revised instructions for authors. 
Finally, we are delighted to recognize the student and resident members of the Annals Editorial Advisory Board who began their service at the November, 2015 meeting.

Nicole Gentile, $\mathrm{PhD}$ will soon receive her MD from the Tulane University School of Medicine. She already is an experienced investigator, and will begin her family medicine residency training at the University of Washington.

Max Romano, MD is a first year resident in family and preventive medicine at the MedStar Franklin Square Medical Center and the Johns Hopkins Bloomberg School of Public Health. He brings strong skills and interests in policy and prevention to his Editorial Advisory Board role.

We welcome your reflections at http://www.Ann FamMed.org.

\section{References}

1. Hendriksen J, Lucassen W, Erkens $P$, et al. Ruling out pulmonary embolism in primary care: comparison of the diagnostic performance of "gestalt" and the Wells rule. Ann Fam Med. 2016;14(3):227-234.

2. Phillips J, Hustedde C, Bjorkman S, et al. Rural women family physicians: strategies for successful work-life balance. Ann Fam Med. 2016;14:(3):244-251.

3. DeVoe JE. Dad's Last Week. Ann Fam Med. 2016;14(3):273-276.

4. Grande D. Sticker shock: the experience of a health care consumer. Ann Fam Med. 2016;14:(3):270-272.
5. Purkaple BA, Mold JW, Chen S. Encouraging patient-centered care by including quality-of-life questions on pre-encounter forms. Ann Fam Med. 2016;14(3):221-226.

6. Gentile N, Johansen ME. Prostate cancer patients' survival expectations with and without their chosen treatment. Ann Fam Med. 2016; 14:(3):iii.

7. Xu J, Janisse J, Ruterbusch J, et al. Patients' survival expectations with and without their chosen treatment for prostate cancer. Ann Fam Med. 2016;14:(3):208-214.

8. Duineveld LAM, van Asselt K, Bemelman W, et al. Symptomatic and asymptomatic colon cancer recurrence: a multicenter cohort study.. Ann Fam Med. 2016;14:(3):215-220.

9. Ricci-Cabello I, Valderas JM, Avery A, Reeves D, Kadam U. Measuring patient safety in primary care: the development and validation of the "Patient Reported Experiences and Outcomes of Safety in Primary Care" (PREOS-PC). Ann Fam Med. 2016;14:(3):253-261.

10. Papadakis $S$, Cole AG, Reid RD, et al. Increasing rates of tobacco treatment delivery in primary care practice: Evaluation of the Ottawa Model for Smoking Cessation. Ann Fam Med. 2016;14:(3): 235-243.

11. Carranza Leon BG, Jensen MD, Hartman JJ, Jensen TB. Selfmeasured vs professionally measured waist circumference. Ann Fam Med. 2016;14:(3):262-266.

12. Sharma AE, Willard-Grace R, Hessler D, Bodenheimer T, Thom DH. What happens after health coaching? Observational study 1 year following a randomized controlled trial. Ann Fam Med. 2016;14:(3): 200-207.

13. Olayiwola J. Racism in medicine: shifting the power. Ann Fam Med. 2016;14:(3):267-269. 